\title{
Perspektiven und Handlungslogiken der Integration von Geflüchteten an beruflichen Schulen: Einblicke aus Sachsen/Deutschland
}

\section{Zusammenfassung}

Am Beispiel einer Studie zur Bildungsintegration von jugendlichen Geflüchteten in Sachsen werden divergierende Akteur*innenperspektiven auf Integration offengelegt. Diese unterschiedlichen Lesarten und damit verbundenen Vorstellungen von Integrationserfolgen resultieren in unterschiedlichen und manchmal widersprüchlichen Praktiken der Integration von Geflüchteten. Divergierende Auffassungen von >Integration s sind nicht allein auf individuelle Einstellungen zurückzuführen, sondern eng mit professionellen und organisationalen Perspektiven und Logiken verknüpft. Diese lassen sich nur begreifen, wenn die entsprechenden Perspektiven auf Integration in ihrem raum-zeitlichen Entstehungszusammenhang interpretiert werden. Auf dieser Basis lassen sich Handlungsempfehlungen für die weitere Entwicklung von Integrationsmaßnahmen ableiten.

Schlagworte: Integration, berufliche Bildung, Deutsch als Zweitsprache, Geflüchtete

\section{Refugees at Vocational Schools in Saxony/Germany: Perspectives and Logics of Integration}

\section{Abstract}

This study on the educational integration of adolescent refugees in Saxony reveals divergent stakeholder perspectives on integration, resulting in different and sometimes contradictory practices of refugee integration. Diverging conceptions of (integration〉 are not solely due to individual attitudes, but are closely linked to professional and organizational perspectives and logics. These can only be understood if the corresponding perspectives on integration are interpreted within the spatio-temporal context of the respective case study. On this basis, recommendations for the further development of integration measures can be derived.

Keywords: integration, vocational education, German as second language, refugees 


\section{Einleitung}

Im Laufe der Jahre 2014 bis 2017 stellten über 1,5 Millionen Menschen einen Erstantrag auf Asyl in Deutschland. Unter ihnen waren 543.068 Minderjährige und 357.265 junge Erwachsene zwischen 18 und 25 Jahren, also im bildungs- und ausbildungsrelevanten Alter (BAMF 2015, 2016, 2017, 2018). Ihre Integration in das deutsche Schulsystem stellt eine Herausforderung dar, die sich durch die Heterogenität der Bundesländer und Regionen in Bezug auf Zuwanderungserfahrungen sowie durch die föderale Verfasstheit des deutschen Bildungssystems sehr unterschiedlich äußert. Die schulische Integration von jugendlichen Asylsuchenden berührt generell Fragen der strukturellen und didaktischen Gestaltung sowie Reflexionen und Entwicklungen im erziehungswissenschaftlichen Diskurs (u.a. Migrationspädagogik, Mehrsprachigkeit, Diversity Education). Zugleich finden die Praktiken der Integration an konkreten Schulstandorten mit ihren spezifischen Lagebedingungen und teils divergierenden Perspektiven von verschiedenen schulischen Akteur*innen statt.

Dieser Artikel stellt Ergebnisse aus einer empirischen Studie vor, die im Jahr 2016 in zwei Beruflichen Schulzentren im Landkreis >Erzgebirgskreis` im Freistaat Sachsen durchgeführt wurde. Zielsetzung der Studie war es, die Unterrichtsund Integrationspraxis für ausländische Schüler*innen zu evaluieren, die ein einjähriges intensives Deutschprogramm mit Elementen der Berufsorientierung an den Beruflichen Schulzentren absolvierten. Auf der einen Seite zeigt sich in der Studie, wie verschiedene Politikbereiche und damit auch politische Regelungen aufeinandertreffen, interagieren und manchmal miteinander kollidieren, vor allem Maßnahmen in den Bereichen Bildung, Arbeitsmarkt, Asyl und Migration. Auf der anderen Seite offenbart sie unterschiedliche Handlungsorientierungen der verschiedenen Akteur*innen, die auf verschiedenen Konzepten von >Integration< basieren. Ebenfalls relevant sind regionale Kontextfaktoren. Für die Frage der Implementierung von Handlungskonzepten und dem Anspruch einer guten und chancengerechten Beschulung sind diese mitzudenken. Dieser Beitrag geht folgenden Leitfragen nach:

- Welche Vorstellungen von >Integration` dokumentieren sich in den Erzählungen der wichtigsten Akteur*innen?

- Wie ist diese Interpretation gerahmt?

- Wie beeinflussen unterschiedliche Interpretationen von >Integration〈 Praktiken der Integration? 
Um diese Fragen zu erörtern, wird zunächst auf Konzepte von >Integration « und ihre Entwicklung eingegangen sowie ein Überblick über den Stand der Forschung zur berufsschulischen Integration gegeben. Abschnitt 3 referiert die formalen Rahmenbedingungen zur schulischen Integration von Geflüchteten. Im vierten Abschnitt werden die Fallstudienregion sowie die Erhebungs- und Auswertungsmethoden vorgestellt, um dann im fünften Abschnitt auf die Ergebnisse unserer Studie einzugehen. Im Fazit werden die Ergebnisse in generalisierter Form diskutiert.

\section{Perspektiven auf Integration und die Bedeutung der schulischen Integration}

Verschiedene Migrations- und Integrationsforscher*innen haben auf die Unmöglichkeit hingewiesen, eine allgemeingültige Definition von >Integration $<\mathrm{zu}$ finden. So bezeichnete Robinson (1998: 118) Integration als ein »chaotisches Konzept«, da der Begriff »von vielen genutzt aber von den meisten unterschiedlich verstanden [wird] « (Übersetzung durch die Autor*innen). Ager und Strang (2008: 167) weisen auf die Notwendigkeit hin, nachvollziehbare und messbare Prüfkriterien zu finden, um >Integration ‘ als normatives Konzept nutzbar für politische und planerische Prozesse zu machen. Nicht von der Hand zu weisen ist, dass bereits die theoretische Konzeptualisierung des Begriffs >Integration « an spezifische politische und gesellschaftstheoretische Auffassungen geknüpft ist. Für diesen Aufsatz soll zunächst auf zentrale, in Deutschland verwendete Definitionen und Kriterien von >Integration` eingegangen werden, um diese dann für den Kontext Schule und Bildung genauer zu beleuchten und Kriterien für die eigene Untersuchung abzuleiten.

\subsection{Integration als soziologisches Konzept}

Insbesondere in systemtheoretischen Ausführungen ist die Unterscheidung zwischen der System- und der Akteur*innenebene zentral, um Integrationsprozesse zu verstehen: Während Systemintegration nach Lockwood (1964: 245) als »relationships between the parts « die Beziehungen von Teilen eines Systems thematisiert, wird soziale Integration auf der individuellen Ebene als »relationships between the actors« definiert. Für den Prozess der Integration sind demnach soziale Strukturen und menschliche Interaktionen von Bedeutung. Der Blick auf Systeme und Organisationen und ihre Strukturen ermöglicht es, organisationale 
Logiken und Zusammenhänge zu erkennen, die nicht notwendigerweise intentional zu Exklusion oder auch Diskriminierungen führen können (beispielsweise institutionelle Diskriminierung) und auch nicht allein auf Einstellungen einzelner Personen zurückzuführen sind (vgl. Gomolla/Radtke 2009).

Der auf dem Integrationsbegriff von Lockwood basierende handlungstheoretische Ansatz von Esser (2000) unterscheidet vier Dimensionen der sozialen Integration, nämlich die Kulturation, die Platzierung, die Interaktion sowie die Identifikation, das heißt die emotionale Hinwendung zu Werten der Aufnahmegesellschaft und deren Integration in das eigene Selbstkonzept. Essers Konzept wird im bundesdeutschen Kontext stark rezipiert. Es orientiert sich insbesondere im letzten Punkt an einem vor allem aus den USA stammenden Konzept der Assimilation und setzt primär auf rational handelnde Akteur*innen.

Stärker an der Handlungspraxis und sozialen Einbettung von Akteur*innen ausgerichtet ist die praxeologische Perspektive nach Bourdieu (1986). Verschiedene Kapitalsorten wirken strukturierend auf gesellschaftliche Teilhabe. Im Rahmen von Migrations- und Integrationsprozessen gewinnt zumeist die Frage an Bedeutung, auf welche Weise ökonomisches, kulturelles, soziales und symbolisches Kapital auf den neuen gesellschaftlichen Kontext des Ankunftslandes übertragen werden können. So können die im Herkunftsland erworbenen Bildungszertifikate im Ankunftsland eine andere Wertigkeit haben oder formal gar nicht anerkannt werden. Auch ein Mangel an sozialen Beziehungen und Kenntnissen und Erfahrungen über die Funktionsweisen des Bildungssystems im Ankunftsland können die Orientierung erschweren. Eine fehlende Einbindung in soziale Netzwerke im Ankunftsland kann zur Folge haben, dass Unterstützung für die eigenen Belange fehlt (vgl. Glorius 2014: 182). Obschon fokussiert auf das Handeln der (migrierenden) Akteur*innen, ist deren Praxis aber weniger als Kalkül zu verstehen, denn als Ausdruck sozialer Verortung und damit auch unreflektierter Selbstverständlichkeiten.

\subsection{Integration im Kontext des 'Eigenen ‘ und des 'Anderen}

Wie aus den bisherigen Ausführungen hervorgeht, wird Integration in handlungstheoretischen Ansätzen wie auch im politischen und öffentlichen Diskurs oft einseitig mit Blick auf die Zugewanderten definiert. Die Ankunftsgesellschaft und ihre Systeme bilden aus dieser Perspektive lediglich die Hintergrundfolie für den Integrationsprozess der Neuankömmlinge, denen spezifische Zugänge zu den relevanten Systemen (wie Schule, Arbeit, Wohnungsmarkt, Gesundheit) eröffnet 
werden. Kritische Migrations- und Integrationsforscher*innen weisen in diesem Zusammenhang auf eine Gleichsetzung der Begriffe >Integration` und >Assimilation ' hin, also die Erwartung einer vollständigen Anpassung der Neuankömmlinge an die Gepflogenheiten der Ankunftsgesellschaft (sowie Akzeptanz des ihnen zugewiesenen Platzes in der Ankunftsgesellschaft) (vgl. Bowskill et al. 2007; Crul/Schneider 2010: 1145; Hersi 2014). Diese Perspektive wird seitens der Erziehungswissenschaft, insbesondere der Migrationspädagogik und Diversity Education, scharf zurückgewiesen, weil die Rahmung sowohl von Assimilation als auch Integration auf der Annahme von defizitären oder zumindest in der Bringschuld stehenden >Anderen $>$ basiert, welche nur durch den Verzicht auf eigene Normen, Werte und Gewohnheiten, besondere Anstrengungen und die Übernahme der Normen und Werte der Aufnahmegesellschaft in eine bestehende Struktur zu integrieren seien. Zudem werden in dieser Perspektive die Veränderlichkeit von Gesellschaften und die Entstehung neuer Identitäten und Zugehörigkeiten, wie beispielsweise hybride Identitäten und transnationale Verortungen, ignoriert.

Die aufnehmende Gesellschaft konstruiert sich in dieser dualen Perspektive als homogene Einheit, die durch Zuwanderung und Heterogenisierung herausgefordert oder gar bedroht ist, und vernachlässigt dadurch bereits vorhandene soziale und kulturelle Unterschiede (Nohl 2010: 17ff.). Integration und die Diskussion um Integration kann insofern auch als ein Prozess der Bestimmung und der Unterscheidung zwischen innen und außen bzw. zwischen der Eigengruppe und den >Fremden` interpretiert werden (Riegel 2009: 24), welcher zu einem Prozess der exklusiven Identifizierung und Essentialisierung beiträgt.

In jüngster Zeit finden sich pragmatische Ansätze für die Rahmung der Integration und Partizipation von Migrant*innen, welche sich eher auf post-moderne Individualisierungs- und Fragmentierungsprozesse beziehen und damit eine Trennung zwischen dem Eigenen und dem >Anderen « überwinden. Das post-migrantische Paradigma verweist auf soziale Aushandlungsprozesse, welche die unumkehrbaren Realitäten von Migrationsgesellschaften mit hoher Diversität und einer damit verbundenen Notwendigkeit der Anpassung regulatorischer Strukturen, Institutionen und politischer Kulturen auf diese neuen Realitäten anerkennen (Foroutan 2015). Übertragen auf pädagogische Fragen beinhaltet dies einen Perspektivwechsel: Nicht die Anpassungsleistungen von Schüler*innen stehen im Zentrum, sondern die Frage, wie sich das System Schule und die darin handelnden Akteur*innen auf verschiedene Erfahrungen, Zugehörigkeiten und Ressourcen aller Schüler*innen einstellen kann und muss. Damit wird eine Brücke zum Begriff der >Inklusion` geschlagen, der darauf abzielt, unterschiedliche aber 
gleichwertige Lebenswege auf der Grundlage formaler Regelungen und struktureller Anpassungen zu berücksichtigen (Georgi 2015: 26; Glorius 2014: 197). Das Konzept der Inklusion impliziert so auch die Anerkennung von Vielfalt und Diversität, unter anderem in Bezug auf Mehrsprachigkeit (Gogolin 2013).

\subsection{Integration und Schule}

Als Resultat der eingangs erwähnten Meta-Analyse von Integrationsstudien durch Ager und Strang (2008) wird Bildung als einer von vier zentralen Indikatoren (neben Arbeit, Wohnen, Gesundheit) für Integration betrachtet. Ihre Integrationsfunktion erhält Bildung in dieser Perspektive vor allem dadurch, dass sie Kenntnisse und Fähigkeiten vermittelt, durch die eine spätere Arbeitsmarktintegration ermöglicht wird. Eine Existenz sichernde Beschäftigung wiederum ermöglicht Migrant*innen konstruktiv und aktiv an verschiedenen Systemen der Gesellschaft teilzuhaben. Zudem wurde die Institution Schule als wichtiger Ort identifiziert, um Kontakte zur Aufnahmegesellschaft zu knüpfen und integrationsrelevante Beziehungen aufzubauen (Ager/Strang 2008: 172).

Als wesentliche Hemmnisse einer Bildungsintegration weisen die von Ager und Strang (2008: 172) untersuchten Studien drei Faktoren aus: 1) mangelnde Kenntnisse des Schulsystems und der Lehr- und Lernmethoden, 2) mangelnde Unterstützung beim Spracherwerb sowie 3) Isolation und Exklusion (z.B. durch Mobbing, Rassismus oder fehlende Kontakte). Teilweise bedingen sich diese Faktoren auch gegenseitig: So gab es Schulen, die spezielle Sprachlernklassen zum beschleunigten Spracherwerb vorhielten; dies wirkte sich jedoch negativ auf die Kontaktmöglichkeiten zu einheimischen Schüler*innen und damit auf die soziale Inklusion aus. Ebenso zeigte sich in der Meta-Analyse die Vielschichtigkeit von Faktoren, die auf die individuelle Lebensqualität und den Integrationserfolg einwirken. Nicht nur die >harten Indikatoren wie Bildung, Arbeit, Wohnen und Gesundheit spielten eine Rolle, sondern auch die Freundlichkeit der lokalen Bevölkerung, die Einbindung in die lokale Gemeinschaft sowie in eine ethnische Community, das Praktizieren vielfältiger Aktivitäten in Gemeinschaft mit den Einheimischen (wie Sport, religiöse und zivilgesellschaftliche oder politische Aktivitäten) sowie die Stabilität und Sicherheit der Lebenssituation. Gerade letzterer Faktor wurde in dem aus der Meta-Analyse abgeleiteten Integrationsmodell von Ager und Strang (2008: 179) neben Sprache und kulturellem Wissen als wesentlicher integrationsfördernder (oder -hemmender) Faktor modelliert. 
Zentrale Aspekte von Integration sind folglich auf verschiedenen Ebenen angesiedelt: Auf einer rechtlichen Ebene, einer administrativen Ebene, auf der Ebene der Logik einer Institution, auf einer professionellen, auf einer sozialräumlichen und auf einer individuellen Ebene (vgl. Studnitz 2011). Konkret für die Schule bedeutet dies, dass Zugangsbeschränkungen und -öffnungen zum Bildungssystem, rechtliche Restriktionen und Ansprüche, institutionelle Hürden und Angebote, professionelle Konzepte und Orientierungen ebenso eine Rolle spielen, wie die Wahrnehmungen, Orientierungen und Praktiken von Schulleitenden, Lehrkräften, Peers und neu zugewanderten Kindern und Jugendlichen sowie der lokalen Umgebung. Die formale Eingliederung in das Schulsystem als Schüler*in ist nur ein (formaler) Anteil von Integration. Handlungspraktisch relevant werden schließlich die Partizipation am Schulgeschehen und an den Angeboten, eine professionelle Haltung von Schulleitung und Lehrkräften gegenüber allen Schüler*innen, die Verpflichtung zur Förderung aller Schüler*innen sowie Partizipation in der Schüler*innenschaft und auch im Sozialraum.

\subsection{Aktuelle Forschungen zur Bildungsintegration von Geflüchteten in Deutschland}

Im Kontext der verstärkten Fluchtzuwanderung seit 2015 sind verschiedene Studien zur Bildungsintegration von Geflüchteten entstanden. Konkret auf die berufliche Bildung fokussiert zeigen sich größtenteils übereinstimmende Befunde. So wird in fast allen Studien darauf verwiesen, dass Struktur und Inhalte der berufsschulischen Ausbildung nicht in vollem Umfang auf die Adressat*innen passen. Zum Beispiel wird auf vorhandene Konzepte wie das >Berufsvorbereitungsjahr für Migranten` (BVJ-M) zurückgegriffen, welches aber nicht unbedingt den Ressourcen und Bedarfen der Schüler*innen entspricht, sondern eher eine kostengünstige Lösung darstellt (vgl. Gag/Schroeder 2014; Vogel/Stock 2017; vbw 2016). Ebenfalls übereinstimmend ist die Feststellung, dass geflüchtete Kinder und Jugendliche sehr heterogene Schulerfahrungen haben, dass es zu einer hohen Fluktuation kommt und die differenten Sprachkompetenzen eine besondere Herausforderung in der Beschulung darstellen (vgl. Bauer/Schreyer 2016; Scheiermann/Walter 2016). Die Mischung von bildungs- und aufenthaltsrechtlichen Belangen, wenn es zum Beispiel um die Zulassung zu Berufspraktika geht, sowie generell die unklare Bleibeperspektive von asylsuchenden Schüler*innen erschweren die organisationale Handhabe und eine kontinuierliche Beschulung (vgl. u.a. Spindler 2016). Teils werden auch lange Anfahrtswege als Probleme 
angesprochen, die auf die besondere Unterbringung von geflüchteten Schüler*innen verweisen (vgl. Speer/Klaus 2015). Ein Mangel an Lehrkräften erschwert in manchen Bundesländern und Regionen die Situation zusätzlich (vgl. Heinrichs et al. 2016).

Insgesamt ist zu konstatieren, dass in den Studien ein problemorientierter Blick dominiert, wobei die jeweiligen Probleme sowohl auf struktureller Ebene als auch auf der Ebene der Schüler*innen thematisiert werden. Auf struktureller Ebene werden ausländerrechtliche, finanzielle und regionale Schwierigkeiten und Besonderheiten sowie an wenigen Stellen die Eingeschränktheit der schulischen Instrumente (vgl. Heinrichs et al. 2016) benannt. Auf der individuellen Ebene geht es zumeist um Probleme und Defizite (Deutschkenntnisse) der asylsuchenden Schüler*innen (vgl. Dietz et al. 2016). Insgesamt fällt auf, dass der Integrationsbegriff und das Integrationsverständnis selten zur Diskussion gestellt werden und teils als reine Anpassungsleistung der Schüler*innen verstanden werden bzw. als Kompensation von Defiziten. Mehrsprachigkeit und mehrsprachige oder bilinguale schulorganisatorische Modelle (vgl. Reich/Roth 2002) werden in den Studien selten thematisiert. Einige Studien befragen geflüchtete Personen selbst, wie beispielsweise eine Studie in Hamburg oder auch kleinere Teilprojekte, die vorrangig von Studierende durchgeführt wurden (vgl. Meyer 2014; Gurski/Rother 2018; Spindler 2016; Metzner et al. 2018).

Setzt man die Ergebnisse der Studien in Bezug zu den oben diskutierten Definitionen und Kriterien von Integration, ist festzustellen, dass nicht wenige Arbeiten einem Verständnis und Ziel von Integration folgen, das eher implizit leitend ist, teils strukturelle Dimensionen umfasst, auf der Ebene der Schüler*innen aber größtenteils einem Assimilationsverständnis von Integration folgt. Die Einbindung in und die Rolle des Sozialraums werden kaum thematisiert.

Die nachfolgend vorgestellte empirische Studie setzt bei diesen Forschungslücken an. Durch relativ offene erkenntnisleitende Fragen (vgl. Einleitung) arbeiten wir heraus, welches Verständnis verschiedene Akteur*innen in der Schule von Integration haben und welche Erwartungen und Handlungen sich daraus ergeben. Dabei interessiert uns, welche Themen und Verantwortungen die Befragten explizit oder implizit ansprechen. Der Fokus der Studie liegt damit auf den Wissensbeständen und Handlungspraktiken der Akteur*innen. 


\section{Rahmenbedingungen der Bildungsintegration von Geflüchteten in Deutschland und Sachsen}

Die Bildungsintegration von Geflüchteten wird durch rechtliche und organisatorische Rahmenbedingungen determiniert. Wie im Folgenden noch darzustellen ist, ist die Frage der Bildungsmöglichkeiten von Geflüchteten in Deutschland nicht eindeutig geregelt und überdies durch die föderale Zuständigkeit für Bildung in 16 verschiedene Systeme fragmentiert. Die Implementierung der jeweiligen Gesetzgebungen ist zudem in sich inkonsistent, da je nach betrachteter Gruppe und Schulart unterschiedliche Herangehensweisen zu beobachten sind.

\subsection{Rechtliche und organisatorische Aspekte der Beschulung von Migrant*innen}

Minderjährige Migrant*innen haben in der Regel hinsichtlich Bildung und Schule die gleichen Rechte und Pflichten wie Kinder ohne Migrationshintergrund. Dies bezieht sich insbesondere auf die allgemeine Schulpflicht, die sich in Deutschland auf neun bzw. zehn Schulbesuchsjahre erstreckt, gefolgt von einer dreijährigen Berufsschulpflicht (Vossenkuhl 2010). Hinsichtlich des Höchstalters für die Schulpflicht bzw. das Schulbesuchsrecht gibt es keine bundeseinheitliche Regelung. In vielen Bundesländern endet die Schulpflicht zum Ende des Schuljahres, in dem der/die Schüler*in 18 Jahre alt wird und zwölf Jahre eine Schule besucht hat.

Neben der Schulbesuchspflicht ist - gerade in Bezug auf asylsuchende Schüler*innen - auch das Schulbesuchsrecht von Relevanz, das in Art. 26 der Allgemeinen Erklärung der Menschenrechte vom 10.12.1948 wie auch im Grundgesetz (GG Art. 1 Abs. 1 und Art. 2 Abs. 1 in Verbindung mit Art. 3 Abs. 1) verankert ist. Das gilt zumindest vom Beginn der Schulpflicht bis zum Erreichen der Volljährigkeit. Nach der Richtlinie 2013/33/EU (Aufnahmerichtlinie, hier besonders Art. 14, Abs. 2) ist der Zugang zum Bildungssystem spätestens drei Monate, nachdem ein Antrag auf internationalen Schutz von einem Minderjährigen oder in seinem Namen gestellt wurde, zu gewähren; Minderjährigen sind bei Bedarf Vorbereitungs- und Sprachkurse anzubieten. Die Umsetzung dieser Richtlinie wird in den Bundesländern unterschiedlich gehandhabt. Aus pragmatischen Gründen erfolgt die Zuordnung zu einer Schule in der Regel erst dann, wenn minderjährige Asylsuchende die Erstaufnahmeeinrichtung verlassen haben und ein vorläufiger ständiger Wohnsitz in einer Kommune zugeteilt wurde (Mercator-Institut 2015: 37). Da es durch die zahlreiche Ankünfte von Asylsuchenden in den Jahren 2015 
und 2016 zu Verzögerungen in der Wohnortzuweisung kam, geht eine UNICEFExpertise von vielfachen Verzögerungen des Schulbesuchs von Geflüchteten aus, die im Extremfall den Verlust eines kompletten Schuljahres bedeuteten (UNICEF 2016).

\subsection{Bildungsbeteiligung und Bildungserfolg von ausländischen und asylsuchenden Schüler*innen in Deutschland}

International vergleichende Bildungsmonitorings weisen immer wieder auf die statistisch ablesbaren schlechteren Bildungschancen von Kindern mit Migrationshintergrund in Deutschland hin, die mit unterschiedlichen Faktoren (u.a. Migrationsalter, Sprache im Elternhaus, ökonomischer Hintergrund, Berufswegplanungen, Rassismus, institutionelle Diskriminierung, unflexible Schulen) begründet werden (Auernheimer 2013; ABB 2016; Baur/Häußermann 2009). ${ }^{1}$ Auch am Übergang in die berufliche Ausbildung haben Jugendliche mit Migrationshintergrund überdurchschnittlich häufig mit Problemen zu kämpfen. So befanden sich nur 30\% aller Bewerber*innen mit Migrationshintergrund zum Ende des Jahres 2016 tatsächlich in einem Ausbildungsverhältnis, während dies bei den Ausbildungsinteressierten ohne Migrationshintergrund jedem/jeder Zweiten gelungen war. Hingegen war der Anteil der Bewerber*innen mit Migrationshintergrund im sogenannten Übergangssystem, aber auch unter den (ungelernt) Erwerbstätigen und Arbeitslosen, deutlich höher als bei den Personen ohne Migrationshintergrund (BiBB 2017: 343).

Im Vergleich $\mathrm{zu}$ anderen Jugendlichen mit Migrationshintergrund haben jugendliche Geflüchtete jedoch vielfach mit weiteren oder gravierenderen Problemlagen zu kämpfen, die die Teilhabe am deutschen Bildungs- und Ausbildungssystem, so wie es aktuell aufgestellt ist, zusätzlich erschweren: beispielsweise können Bildungsdefizite existieren, wenn sie durch die Flucht über lange Zeit am Schulbesuch gehindert waren oder in ihrem Herkunftsland keinen Zugang zu weiterführenden Schulen hatten. Eine zweite Barriere stellen die fehlenden Deutschkenntnisse dar, was sich zusammen mit Lücken des Schulbesuchs insbesondere im fortgeschrittenen Bildungsalter im deutschen, monolingual ausgerich-

1 Ein nachhaltiges Problem für die empirischen Analysen in diesem Bereich bildet die Tatsache, dass die verschiedenen statistischen Erhebungen unterschiedliche soziale Merkmalsdefinitionen nutzen. Während manche Studien zwischen In- und Ausländer*innen unterscheiden, erfassen andere Studien einen sogenannten Migrationshintergrund. Zur Problematik der Operationalisierung siehe auch Kemper (2017). Zur kritischen Auseinandersetzung mit dem Begriff des Migrationshintergrundes: El-Mafaalani (2016). 
teten Schulsystem als gravierend erweist, da dann nicht mehr viele Schulbesuchsjahre verbleiben, in denen dieser Rückstand aufgeholt werden kann.

Somit sind gerade jugendliche Geflüchtete zwischen 15 und 18 Jahren stark auf Vorbereitungskurse im sogenannten Übergangssystem angewiesen, die einen Sprachlehrgang mit berufsorientierenden Elementen und gegebenenfalls mit dem Nachholen von allgemeinschulischen Bildungsinhalten verbinden (MercatorInstitut 2015; Schroeder et al. 2015). In einigen Bundesländern endet die Berufsschulpflicht mit Vollendung des 18. Lebensjahres, sodass dann nur noch die Möglichkeit verbleibt, außerschulische Sprach- und Integrationskurse zu belegen, um damit die Ausbildungsreife zu erlangen. Häufig sind diese jedoch nicht verzahnt oder aus formalen oder logistischen Gründen schwer zugänglich (vgl. Baethge/ Seeber 2016).

Weitere Hemmnisse beim Übergang von geflüchteten Jugendlichen in die berufliche Ausbildung können mangelnde Kenntnisse und Informationen über das duale Ausbildungssystem darstellen, da dieses in den Herkunftsländern in der Regel nicht existiert und in seiner Reichweite gegebenenfalls nach Ankunft in Deutschland auch nicht vermittelt und hinreichend verstanden wird. Die Jugendlichen - insbesondere, wenn sie allein als unbegleitete minderjährige Flüchtlinge eingereist sind - sind in diesem Fall stark auf die Unterstützung sozialer Netzwerke der Ankunftsgesellschaft angewiesen, um die Optionen des berufsbezogenen Ausbildungssystems für sich zu erschließen und anzuwenden.

Auch ein aufenthalts- oder sozialrechtlich relevanter Statuswechsel im Verfahren (z.B. die Zuerkennung eines bestimmten Aufenthaltsstatus oder das Erreichen der Volljährigkeit bei unbegleiteten minderjährigen Geflüchteten) kann ein zusätzliches Problem darstellen. Das gilt, wenn ein anderer Aufenthaltsort zugewiesen wird, was die Zugänglichkeit zu laufenden Bildungsmaßnahmen erschwert. Darüber hinaus kann es geschehen, dass laufende Bildungsmaßnahmen durch einen formalen Statuswechsel nicht mehr besucht werden dürfen. In der Auslegung dieser rechtlichen Aspekte sind Handlungsspielräume enthalten, die von den zuständigen Behörden durchaus unterschiedlich genutzt werden (dies zeigt aktuell die hohe Variabilität bei der Gewährung von sogenannten Ausbildungsduldungen). 


\subsection{Struktur und Entwicklung der schulischen Bildungsangebote für Migrant*innen im Freistaat Sachsen}

Im Bundesland Sachsen werden Schüler*innen mit Migrationshintergrund, die noch keine oder nur geringe Deutschkenntnisse aufweisen, zunächst separat in Vorbereitungsklassen unterrichtet, um sie dann Schritt für Schritt in den Regelunterricht zu integrieren. Diese Praxis basiert auf der Sächsischen Konzeption zur Integration von Migranten (SMK 2000). Seit dem Schuljahr 2010/11 wird der sächsische Lehrplan Deutsch als Zweitsprache flächendeckend umgesetzt. Allerdings hatte der Freistaat Sachsen aufgrund geringer Zuwanderungszahlen lange Zeit eine nur schwach entwickelte Infrastruktur für die Integration von ausländischen Schüler*innen. Die Zuwanderung aus dem Ausland war sehr moderat, der Ausländeranteil lag bis zum Jahr 2014 bei unter 3\%, und gerade in den ländlichen Regionen gab es kaum Bedarf an schulischen Integrationsangeboten. Die meisten Vorbereitungsklassen befanden sich an Grund- und Mittelschulen; nur wenige Vorbereitungsklassen wurden an beruflichen Schulen oder Schulen des zweiten Bildungsweges (Abendschule, Kolleg) eingerichtet.

Dies änderte sich mit dem Anstieg des Zuzugs von Asylsuchenden im Jahr 2015, der zu einem rasch steigenden Bedarf an schulischen Integrationsangeboten führte: Im Laufe des Jahres 2015 wurden 69.900 Asylsuchende in Sachsen registriert. In den vorherigen Jahren waren die Zahlen von unter 3.000 jährlich ab 2011 allmählich bis auf 11.786 im Jahr 2014 angestiegen (vgl. DSA 2018: 138). Die paritätische Verteilungspraxis bedingte, dass insbesondere ab dem Schuljahr 2015/16 überall im Freistaat schulische Integrationsangebote ausgebaut werden mussten. Während des ersten Schulhalbjahres 2015/16 hatte sich die Anzahl der Schüler*innen in Vorbereitungsklassen mehr als verdoppelt (von 3.751 auf 7.531). Die Anzahl von vorbereitenden Klassen stieg von $290 \mathrm{zu}$ Beginn des Schuljahres auf 469 im März 2016. Davon befanden sich 189 Klassen an Grundschulen, 151 an Gymnasien und 122 an beruflichen Schulen (Kelch 2016).

Eine Betrachtung der Entwicklung in den Schuljahren 2012/13 bis 2016/17 zeigt den starken Ausbau der schulischen Integrationsangebote und auch den Aufwuchs der Schüler*innen (Abbildung 1). Während im Schuljahr 2012/13 insgesamt 126 Vorbereitungsklassen existierten, davon $12,5 \%$ an beruflichen Schulen, waren es im Schuljahr 2016/17 568 Vorbereitungsklassen, davon 30\% an beruflichen Schulen. Die Schülerzahl in den Vorbereitungsklassen an beruflichen Schulen verzehnfachte sich im Betrachtungszeitraum, von 215 auf 2.112 Schüler*innen. 
Abbildung 1: Vorbereitungsklassen nach Schulart in Sachsen, Schuljahr 2012/13 bis Schuljahr 2016/17

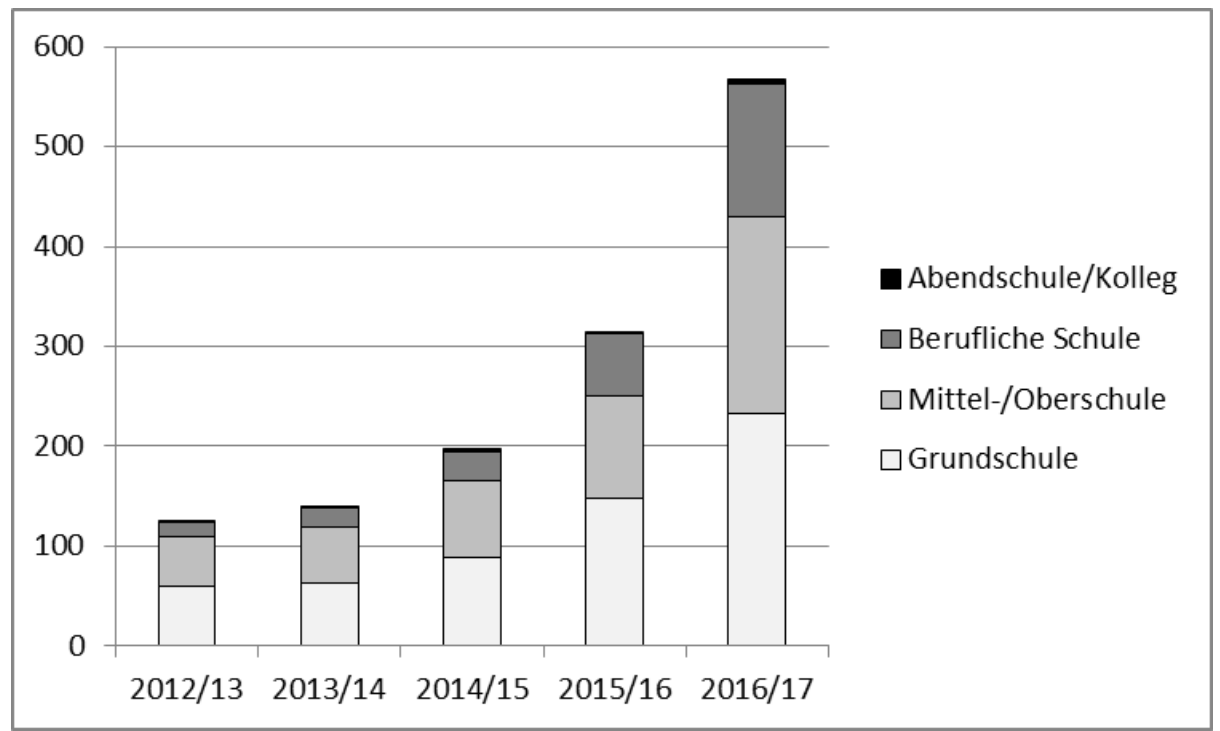

Quelle: Statistisches Landesamt Sachsen, eigener Entwurf

Anders als die Vorbereitungsklassen an allgemeinbildenden Schulen, bei denen ein gradueller Übergang der ausländischen Schüler*innen in die Regelklassen vorgesehen ist, sind die >Vorbereitungsklassen mit berufspraktischen Aspekten an den Beruflichen Schulzentren als berufsvorbereitende Angebote konzipiert: Die Schüler*innen erhalten Unterricht im Fach Deutsch als Zweitsprache. Zudem sollen sie »zwei Monate am fachtheoretischen und fachpraktischen Unterricht an Beruflichen Schulzentren entsprechend dem individuellen künftigen Ausbildungsbereich teil[nehmen]« (SMK 2000: 1). Während des Schuljahres 2015/16 wurde diese Konzeption in leicht abgewandelter Form praktiziert: 24 Unterrichtstunden Deutsch als Zweitsprache pro Woche werden flankiert von sechs Stunden fachpraktischem Unterricht, was den Schüler*innen einen Überblick über Berufs- und Ausbildungsfelder verschaffen soll. Die fachpraktischen Einheiten, so der Plan, sollen integrativ, das heißt in Form stundenweiser Integration der ausländischen Schüler*innen in eine Berufsschulklasse, durchgeführt werden (Interview LT1).

Als Reaktion auf die große Zahl der ankommenden Geflüchteten, die schulpflichtig waren oder ein Schulbesuchsrecht hatten, wurde im Laufe des Schuljahres 2015/16 eine Reihe von Maßnahmen ergriffen, um trotz knapper Ressourcen 
Bildungsangebote zu schaffen. Während zu Beginn des Schuljahres im August 2015 auch über 18jährige Asylbewerber*innen in Vorbereitungsklassen mit berufspraktischen Aspekten aufgenommen wurden, wurde diese Regelung im zweiten Schulhalbjahr 2015/16 aufgrund mangelnder Kapazitäten aufgegeben (SMK 2016). Daraus folgte, dass Schüler*innen, die während des Schuljahres volljährig wurden, die Vorbereitungsklassen verlassen und sich selbstständig um einen Platz in einem der Integrationskurse bemühen mussten, welche in der Zwischenzeit vom Bundesamt für Migration und Flüchtlinge und der Agentur für Arbeit eingerichtet worden waren. Zudem wurden verschiedene Modellprojekte konzipiert, die sich auf die Begleitung des Übergangs von der Schule in die Ausbildung richteten.

In der Betrachtung der Rahmenbedingungen der Bildungsintegration von Geflüchteten in Deutschland sehen wir aus systemtheoretischer Sicht, wie über verschiedene Organisationen sowie politische Maßnahmen Inklusions- und Exklusionsmechanismen konstituiert werden, die jeweils an spezifische Bedingungen geknüpft sind (Sprachkenntnisse und Schulbesuch). Zieht man die Theorie Bourdieus zu Kapitalsorten hinzu, erweitert sich die Perspektive auf die Frage, wie Personen überhaupt in die Lage kommen, bestimmte Erwartungen einer Organisation erfüllen zu können und auch, wie sie sich zu den Regeln und Vorschriften verhalten.

\section{Kontext, Methodik und Durchführung der empirischen Studie}

Die empirische Basis für diesen Artikel stammt aus einer Studie, die die Unterrichts- und Integrationspraxis für ausländische Schüler*innen evaluierte, welche ein einjähriges intensives Deutschprogramm mit Elementen der Berufsorientierung an den Beruflichen Schulzentren absolvierten. Im Folgenden stellen wir die Erhebungsregion mit ihren strukturellen Kontextbedingungen vor und geben Auskunft zu den Erhebungs- und Auswertungsmethoden.

\subsection{Region und Rahmenbedingungen}

Die Studie wurde an zwei Beruflichen Schulzentren im Landkreis >Erzgebirgskreis $<$, einer peripheren Mittelgebirgsregion an der Grenze zur Tschechischen Republik, durchgeführt. Der Landkreis ist dünn besiedelt und leidet, wie viele andere ländliche Regionen, seit Jahrzehnten unter Bevölkerungsverlust. Vor allem jüngere Erwachsene verlassen die Region zum Studium oder zu Ausbildungszwe- 
cken und siedeln sich anschließend in größeren Städten an, die als attraktiver erscheinen. Der andauernde Bevölkerungsverlust führte unter anderem zu einem Rückbau der Bildungsinfrastruktur, was im Bereich der beruflichen Bildung durch die Zusammenlegung von vormals eigenständigen beruflichen Schulen zu Beruflichen Schulzentren realisiert wurde. Wirtschaftlich ist die untersuchte Region relativ stark: Der traditionelle Bergbau hat den Grundstein für verschiedene Branchen des produzierenden Gewerbes gelegt, das vorwiegend klein und mittelständisch organisiert ist. In Bezug auf die weitere demographische Entwicklung im Landkreis gibt es ernsthafte Bedenken hinsichtlich der zukünftigen Fachkräfteverfügbarkeit (Wirtschaftsförderung Erzgebirge 2014: 35). Entsprechend wird der beruflichen Ausbildung und der Verfügbarkeit von berufsbildenden Angeboten eine große Bedeutung für die zukünftige Entwicklung der Region beigemessen.

\subsection{Durchführung der Studie}

Die Studie wurde im Jahr 2016 durchgeführt und spiegelt vor allem das Schuljahr 2015/2016, das stark beeinflusst war durch die große Zahl der Ankünfte im Sommer 2015 und die damit verbundenen Reaktionen. Dies ist insofern für die Studie von Bedeutung, als dass die Erhebungen in einer Phase stattfanden, in der Gemeinden, Behörden und Schulen gerade erst begannen, ihre Integrationsansätze und -praktiken (s. oben) zu systematisieren bzw. sich darauf einzustellen, dass sie es mit einem länger andauernden Phänomen zu tun haben. Die Datenerhebung fand zwischen Februar und Juli 2016 in zwei (von insgesamt drei) Beruflichen Schulzentren des Erzgebirgskreises statt. Die beiden untersuchten Schulzentren verteilen sich auf fünf Standorte, mit einer Entfernung von 20 bis $45 \mathrm{~km}$ zwischen den Schulteilen. Beide Berufliche Schulzentren bieten eine Vielzahl von schulischen und beruflichen Bildungsgängen an. Vor allem mit den berufsschulischen Fachklassen werden die Bedarfe der Betriebe in der Region gedeckt, mit Bildungsgängen z.B. im Bereich Bau, Gastronomie, Holzbearbeitung, Elektronik und Elektrotechnik, KfzTechnik, Mechatronik, Metallbau sowie in Kaufmännischen und Verwaltungsberufen. Hinzu kommen Bildungsgänge zur Erlangung allgemeinbildender Schulabschlüsse (Berufsbildendes Gymnasium, Fachoberschule) und berufsvorbereitende Bildungsgänge (Berufsvorbereitungsjahr, Einstiegsqualifizierung).

Das eine Berufliche Schulzentrum hatte zu Beginn des Schuljahres 2015/16 954 Schüler*innen (unter ihnen 53 Asylsuchende in vorbereitenden Klassen), das andere 1.717 - und 73 Asylsuchende in vorbereitenden Klassen (im Folgenden VBK-Schüler*innen genannt), vor allem aus Afghanistan, Eritrea, Syrien und 
dem Kosovo. Während des Schuljahres erhöhte sich die Zahl der VBK-Schüler*innen auf 72 bzw. 108. Insgesamt herrschte eine relativ hohe Fluktuation unter den VBK-Schüler*innen, die sich aus Änderungen im Asylstatus, aus der Zusammenlegung von Unterkünften und damit einhergehender Schulwechsel, oder aus Veränderungen der gesetzlichen Rahmenbedingungen für den Schulbesuch ergab. Die Schüler*innen wohnten in Gruppenunterkünften oder in ihren Familien in den umliegenden Ortschaften. An einem der Schulstandorte befand sich zudem eine Gruppenunterkunft für unbegleitete minderjährige Geflüchtete, die die Vorbereitungsklasse des Beruflichen Schulzentrums besuchten.

Die eingesetzten Forschungsmethoden umfassten Vor-Ort-Begehungen, informelle Gespräche mit den verschiedenen Akteur*innengruppen, teilnehmende Beobachtung sowie Gruppendiskussionen und leitfadengestützte Interviews. Zielsetzung war die Erfassung des Status Quo aus der Sicht der handelnden Akteur*innen, um Handlungsorientierungen und -möglichkeiten zu identifizieren. Die Befragung umfasste an den ausgewählten Beruflichen Schulzentren beide Schulleiter, einen stellvertretenden Schulleiter sowie sieben Lehrkräfte in Vorbereitungsklassen und drei Gruppen von VBK-Schüler*innen. Zusätzlich wurden Expert*inneninterviews mit zwei weiteren relevanten Personen im Bereich Koordination für Migration und Integration und Berufsschule geführt. Um einen vielseitigen Überblick zur regionalspezifischen Situation und um weiterführende Betrachtungen zur berufspraktischen Qualifikation $\mathrm{zu}$ erhalten, fanden Expert*innengespräche mit den relevanten Vertreter*innen der regionalen Industrie- und Handelskammer, der Handwerkskammer, Bundesagentur für Arbeit sowie der Informations- und Beratungsstelle Arbeitsmarkt Sachsen statt. Von den Begehungen und Hospitationen liegen ausführliche Protokolle und Fotodokumentationen vor. Die Interviews und Gruppendiskussionen wurden elektronisch aufgezeichnet und im Anschluss teiltranskribiert, die Namen der Befragten mit Codierungen anonymisiert (vgl. Tabelle 1).

\section{Tabelle 1: Übersicht der erhobenen Daten und Codierungen}

Erhebungsform und Akteur*innengruppe

Protokolle von Begehungen

Protokolle von Hospitationen/teilnehmender Beobachtung

Interviews mit Schulleitern

Interviews mit Lehrkräften

Gruppendiskussionen mit VBK-Schüler*innen

Interviews mit Vertreter*innen der Schulbehörde

Sonstige Expert*inneninterviews

Quelle: eigene Ergebung

$\begin{array}{cc}\text { Anzahl } & \text { Code } \\ 5 & \text { BG } \\ 5 & \text { TB } \\ 2 & \text { LT } \\ 9 & \text { LK } \\ 3 & \text { SL } \\ 2 & \text { SBA } \\ 4 & \text { EX }\end{array}$




\subsection{Auswertungsmethodik}

Das Datenmaterial wurde auf Grundlage der Dokumentarischen Methode (Bohnsack 2014) ausgewertet, die ihren Ursprung in der Wissenssoziologie hat (vgl. Mannheim 1964). Dieser Ansatz ermöglicht einen Zugang zu impliziten, kollektiv geteilten Wissensbeständen, in die das Handeln eingebettet ist, ohne dass dies den Handelnden explizit bewusst ist (Mannheim 1964). Die Dokumentarische Methode zielt darauf ab, kollektive Orientierungen und den >Modus Operandi< des spezifischen Handlungsfeldes herauszuarbeiten. Das erhobene Material wird dabei einem mehrstufigen Interpretations- und Abstraktionsprozess unterzogen. Während in einem ersten Schritt der immanente Sinn des Erzählten mit seinen subjektiven Deutungen, Einstellungen und Alltagstheorien rekonstruiert wird, konzentriert sich der zweite Analyseschritt auf den Dokumentsinn des Gesagten, das heißt auf die kollektiven Handlungsorientierungen, die dem Handeln zugrunde liegen (vgl. Przyborski/Wohlrab-Sahr 2014: 20). Die hier angewandte Interpretationsstrategie fokussierte darauf, die Einschränkungen sowie Handlungsoptionen und die entsprechenden Handlungspotenziale aus der Perspektive der Interviewpartner*innen herauszuarbeiten. Durch die Kontrastierung der verschiedenen Orientierungen, geteilte und auch differente, lassen sich (habitualisierte) Praktiken (s. oben) und deren Begründung offenlegen, insbesondere auch in Bezug auf formale Regeln einer Organisation (vgl. Bohnsack 2017; Nohl 2017).

Die vergleichende Analyse ermöglicht es unter anderem, unterschiedliche Perspektiven auf den Schlüsselbegriff der Integration zu erkennen, die kontextuelle Einbettung der eigenen Handlungsorientierung zu untersuchen sowie den Einfluss unterschiedlicher Lesarten von Integration auf die Handlungspraxis zu identifizieren. Dabei wurden die Aussagen der Befragten nicht primär als individuelle oder persönliche Ansichten zum Thema verstanden, vielmehr vermitteln sie konkretes und kollektiv geteiltes Wissen einer spezifischen Praxis und sie zeigen auf, wie typischerweise in den Beruflichen Schulzentren mit dem Thema Integration umgegangen wird. Die Erfassung dieser subjektiven Akteur*innen-Perspektive ist aus zwei Gründen von Bedeutung: Zum einen, weil sich aus der subjektiven Problem- und Situationswahrnehmung das individuelle Handeln vor Ort ableitet. Zum anderen, weil sich durch eine vergleichende Analyse der subjektiven, akteur*innenspezifischen Lagebeurteilung wichtige Erkenntnisse hinsichtlich der Wirksamkeit von normativen Handlungsgrundlagen ableiten lassen. Diese sind zugleich ein wichtiger Baustein bei der Beurteilung der Wirksamkeit von Maßnahmen und der Ableitung von Handlungsempfehlungen. 


\section{Ergebnisse}

Ausgehend von den Analysen der empirischen Daten konnten drei Perspektiven auf Integration unterschieden werden: Zum einen eine systemorientierte Lesart, in der organisatorische Aspekte hervorgehoben wurden, zum zweiten die sprachliche Integration und zum dritten die soziale Integration in und außerhalb der Schule. Die Gewichtung dieser drei Elemente von Integration war je nach Akteur*innengruppe unterschiedlich.

\subsection{Integration als organisatorische Herausforderung und die Frage der Zuständigkeit}

Aus einer organisatorischen Sicht war die Integration von asylsuchenden Schüler*innen im Schuljahr 2015/16 eine Koordinierungsaufgabe für die jeweiligen Schulen, die zum Zeitpunkt der Erhebung noch nicht abgeschlossen war. Aus der Perspektive der Schulleitung war die Bereitstellung von Vorbereitungsklassen und deren Integration in die Schulstruktur dabei die Hauptaufgabe. Die Schulleiter der untersuchten Beruflichen Schulzentren konzentrierten sich vor allem auf materielle Aspekte in der Vorbereitung dieser Klassen, wie die Anschaffung von Schulbüchern, Wörterbüchern und spezieller Ausrüstung für den praktischen Unterricht, zum Beispiel Schutzbrillen für das Schweißen. Abgesehen davon wurden mögliche Auswirkungen der Etablierung von Vorbereitungsklassen am Schulstandort auf das soziale Gefüge der Schule - wie etwa rassistische Spannungen oder Konflikte über knappe (Zeit)Ressourcen - eher nicht in Betracht gezogen. So kam es, dass weder die anderen Schüler*innen oder Lehrkräfte auf die Anwesenheit der Geflüchteten - etwa durch eine interkulturelle oder antirassistische Sensibilisierung - eingestimmt wurden, noch die schrittweise Integration der Geflüchteten in Regelklassen konkret vorbereitet und in die Unterrichtsplanung für die Regelklassen integriert wurde.

Die Lehrkräfte der Vorbereitungsklassen fühlten sich allein gelassen bei allen Aspekten der Integration, in der sie auf die Zusammenarbeit mit anderen Lehrkräften angewiesen waren. Dies betraf zum Beispiel die schrittweise Integration von geflüchteten Schüler*innen in den praktischen Unterricht der BerufsschulFachklassen (was oft von Lehrer*innen mit dem Hinweis auf knappe Kapazitäten und einer Priorisierung auf die Prüfungsvorbereitung der >regulären` Auszubildenden abgelehnt wurde), die Vermittlung der Geflüchteten in Praktika (dies hätte zusätzliches Engagement des Schulpersonals notwendig gemacht) oder praktische Fragen in Bezug auf den (integrativen) Sportunterricht (Problematisierung von 
gemischtgeschlechtlichem Unterricht oder mangelnder Sportausrüstung). In Abwesenheit einer top-down-Planung und innerhalb eines allgemein eher reservierten Klimas behalfen sich die Lehrkräfte der Vorbereitungsklassen mit individuellen Absprachen mit einzelnen aufgeschlossenen Lehrkräften, auch wissend, dass andere sich weigerten, überhaupt »Ausländer« (Zitat) zu unterrichten. »Dann gehe ich den Weg des geringsten Widerstandes und suche mir die Leute, wo ich genau weiß, die bemühen sich, sie mit einzubeziehen, denn das ist nicht immer leicht« (LK5, Z. 613-615). Auch in weiteren Einlassungen der Lehrkräfte in den Vorbereitungsklassen kam immer wieder zum Vorschein, dass sie sich und ihre Arbeit im Vergleich zum restlichen Kollegium als marginalisiert wahrnahmen (bis hin zur Bezahlung) und dass die Integration der Geflüchteten nicht als gemeinsames Projekt verstanden wurde.

Zum Ende des Schuljahres wurde die Frage nach dem weiteren Bildungsweg für die Geflüchteten immer virulenter. Während die Schüler*innen manchmal sehr hohe Bildungsaspirationen zum Ausdruck brachten und die anwesenden Forscherinnen über mögliche Zugänge in die Hochschulausbildung befragten, waren Lehrkräfte und Vertreter*innen der Schulbehörde weniger optimistisch, was die künftigen Perspektiven der Schüler*innen anging. Sie gingen davon aus, dass die Schüler*innen aufgrund mangelnder Deutschkenntnisse, aber auch allgemeiner Kenntnisse und Fähigkeiten, weitere pädagogische Unterstützung benötigen würden, um die Ausbildungsreife zu erlangen. Verschiedene Probleme entstanden durch die mangelnde Passfähigkeit der Regularien für den Arbeitsmarktzugang mit den Bildungszielen der Vorbereitungsklassen: Für die meisten Ausbildungsplätze werden ein allgemeinbildender Schulabschluss und ein Deutschzertifikat benötigt; beides konnte in den Vorbereitungsklassen aber nicht erlangt werden. Dies wurde auch von einer verantwortlichen Lehrkraft kritisiert, die davon ausgeht, »dass wir sozusagen auf Halde produzieren, die haben zwar Deutsch gelernt, aber sie können dann damit nichts anfangen« (LK5, Z. 1251-1252).

Auch die Vorschriften über den Zugang zum sogenannten Übergangssystem bildeten ein Hindernis für die Fortsetzung der Schulbildung und die Erlangung eines Schulabschlusses: Eine Reihe von Orientierungs- und Vorbereitungsprogrammen, die für Jugendliche ohne sekundären Abschluss entwickelt wurden (z.B. Berufsgrundbildungsjahr, Einstiegsqualifizierung), sind verknüpft mit dem Schulpflichtalter, was bedeutet, dass Schüler*innen über 18 Jahre nicht in diese Klassen aufgenommen werden. Dies betrifft auch die Vorbereitungsklassen: Während des laufenden Schuljahres wurde eine Verordnung durchgesetzt, die Schüler*innen mit dem Erreichen des 18. Lebensjahrs den Zugang zu den Vorbereitungsklassen mit berufspraktischen Aspekten verwehrte, begründet mit steigenden 
Flüchtlingszahlen und mangelnden Kapazitäten, aber auch mit der mangelnden Passfähigkeit des VBK-Programms mit den vermuteten Bedürfnissen der jungen Geflüchteten sowie mit mangelnder Zuständigkeit:

»Für diese Zielgruppe der jungen Menschen mit Migrationshintergrund sind vorrangig Deutsch-Sprachkurse vonnöten, die ihnen eine schnelle Orientierung in den unterschiedlichen gesellschaftlichen Bereichen erlauben. Darüber hinaus bedarf es zielgerichteter und unmittelbarer Zugänge zu Unternehmen und zum deutschen Arbeitsmarkt. Diese Maßnahmen liegen nicht im Zuständigkeitsbereich des SMK [Sächsisches Ministerium für Kultus] (SMK 2016: 1).

Stattdessen wurde den asylsuchenden Schüler*innen empfohlen, sich um einen Sprachkurs als Teil von Integrationsmaßnahmen zu kümmern, die von verschiedenen Akteur*innen wie der Agentur für Arbeit oder dem Bundesamt für Migration und Flüchtlinge angeboten werden. Diese Verordnung, die zu Beginn des zweiten Schulhalbjahres 2015/16 eingeführt worden war, wurde von Lehrkräften der geflüchteten Schüler*innen stark kritisiert, sahen sie doch ihr Bemühen um Integration damit als vergeblich an:

»Ich hatte Leute, die erst im Februar begonnen haben und die mussten alle raus. Ich habe bis zum letzten Schultag, habe ich dort Anträge im Amt laufen gehabt [...] das haben die Leute im Amt an irgendeiner Position, die mit dieser Arbeit und diesen Jugendlichen überhaupt nichts zu tun haben, entschieden. Die haben entschieden, kommen nicht mehr rein, es ist kein Platz für sie. So und die sollen dann alle über Integrationskurse laufen. Die Integrationskurse kriegen die aber nur, wenn der Status geklärt ist und wer eben eine Aufenthaltsgestattung hat und der Asylantrag bearbeitet wird oder eine Duldung, die haben kein Recht dazu« (LK5, Z. 94-107).

Die restriktiven Regelungen werden umso mehr mit Unverständnis betrachtet, als eine Ausbildungsintegration der Geflüchteten in der durch das produzierende Gewerbe und Handwerksbetriebe geprägten Region grundsätzlich als realistisch und wünschenswert erscheint. Die Gesprächspartner*innen betonten die nachhaltige Gewinnung von Arbeitskräften als essentiell für die wirtschaftliche Entwicklung der Region und betrachteten die jungen Geflüchteten vor diesem Hintergrund als mögliche Personalressource. Die Integration von jungen Migrant*innen im Bildungswesen und auf dem Arbeitsmarkt wird damit als ein zentrales Ziel der Integrationsbemühungen angesprochen. Jedoch, darauf weisen die Befragten hin, kann dieses Ziel nur mit großer, gemeinsamer Anstrengung aller relevanten Akteur*innen erreicht werden.

»Dass diese, eh diese Lücke schließt und man im Prinzip alle Spieler auf dem Brett an einem Strang ziehen, also dass die nicht irgendwo nach der Vorbereitungsklasse im Sankt Nimmerlein verschwinden, sondern wenn sie hier sind, dass man sie im System behalten 
und dann letztlich mal zu einer Fachkraft werden, denn das soll das Ziel, das muss es auch eigentlich sein.«(SBA2, Z.256-262)

Konkretes Beispiel dafür sind die Versuche, junge Menschen aus den Vorbereitungsklassen in den Bereich der Metallbearbeitung einzuführen, da es hier eine starke Nachfrage nach qualifizierten Arbeitskräften und dementsprechend eine hohe Motivation der relevanten Akteur*innen gibt, die jungen Asylsuchenden für eine betriebliche Ausbildung zu gewinnen.

»Unser Ziel ist immer wieder, wir wollen ja gerne, dass wir die bissel wecken für die Metalltechnik, das vielleicht einer oder zweie sagen, ich könnte mir vorstellen, später mal in dem Beruf zu arbeiten in Richtung Metall. Denn das würde gesucht im Erzgebirge, die sind da alle heiß drauf und suchen Lehrlinge in der Metalltechnik, also auch hier wie gesagt Riesenbedarf« (LT2, Z.593-597).

Das oben angesprochene Problem von fehlenden Bildungs- und Sprachzertifikaten könnte nach Ansicht der Gesprächspartner*innen in Zusammenarbeit mit den Kammern und Arbeitgeber*innen gelöst werden (z.B. durch Lockerung der formalen Zugangsvoraussetzungen für eine duale Ausbildung). Ein entscheidender Erfolgsfaktor wäre zudem aus Sicht der Interviewten der direkte Kontakt zwischen den Geflüchteten und potenziellen Arbeitgeber*innen, der durch die Unterstützung von vermittelnden Akteur*innen aufgebaut werden müsste: »da müssen sich, ich sag mal, äh, Handwerksmeister und der Lehrling wirklich finden, dass das funktioniert « (SBA2, Z. 217-219).

Theoretisch verfügen beide untersuchten Beruflichen Schulzentren über umfangreiche Netzwerke, da sie über die Berufsschulklassen Kontakte zu den meist kleinen und mittelständischen Betrieben in der Region besitzen. Allerdings zeigte die Untersuchung, dass sich bisher weder die Schulleiter noch die Lehrkräfte für eine Aktivierung der Netzwerke verantwortlich gefühlt haben. Vielmehr wünschten sie sich zusätzliches Personal sowie einen konkreten Handlungsauftrag für die Realisierung dieser Aufgabe. Diese abwartende Haltung wurde stets durch die langjährigen und teils frustrierenden Erfahrungen im System der Bildungspolitik und -planung begründet. Die Gesprächspartner*innen monierten, dass Bildungspolitik und -planung >von oben< kaum auf die konkreten Bedürfnisse vor Ort eingehe. Andererseits hätten individuelle Akteur*innen vor Ort (die Lehrkraft/die Schulleitung), die diese konkreten Bedürfnisse kennen und Handlungsstrategien und -potenziale sehen, keinerlei Eingriffsmöglichkeiten. Auf diese Weise scheitern mögliche organisatorische Schritte, um den Übergang junger Geflüchteter von der Vorbereitungsklasse in Praktika oder Ausbildungsverhältnisse zu ermöglichen, an der subjektiv wahrgenommenen Hürde der fehlenden Zuständigkeit seitens der handelnden Akteur*innen. 


\subsection{Spracherwerb als Grundlage der Integration}

Der Erwerb deutscher Sprachkenntnisse auf bildungssprachlichem Niveau ist das zentrale Ziel der einjährigen Vorbereitungsklassen mit berufsbildenden Aspekten. Damit wird der Spracherwerb in das Zentrum des Integrationsprozesses gestellt, aus dem heraus sich weitere Integrationsschritte (Arbeitsmarktintegration, gesellschaftliche Integration etc.) ergeben können. Anders als bei der Integration im allgemeinschulischen Bereich wird der Pflege der Herkunftssprache hier keine Bedeutung beigemessen. Obgleich viele der Schüler*innen nicht nur eine Herkunftssprache beherrschen, sondern zum Teil multiple Sprachkenntnisse besitzen (die vielfach auch ihren bisherigen Migrationsweg repräsentieren), wird auf diese Kompetenzen während des Unterrichts praktisch gar nicht eingegangen. Damit ist die Übertragung kulturellen Kapitals - und seine Wertschätzung - in diesem Setting nicht gewährleistet.

Die Kompetenzen der Schüler*innen wurden von den Lehrkräften als sehr heterogen beschrieben, nicht nur in Bezug auf ihr Sprachlernvermögen, sondern auch hinsichtlich der bisherigen Bildungserfahrungen und -kompetenzen, welche zwischen nahezu Hochschulzugangsberechtigung und Analphabetismus lagen. Diese Heterogenität forderte von Lehrkräften, flexibel zwischen verschiedenen Qualifikationsniveaus zu wechseln und stellte für weiter fortgeschrittene Schüler*innen eine Beeinträchtigung des Lernfortschritts dar.

»Das geht nicht auf Tempo, eine Sprache lernen. Das wissen sie auch, sie sagen selber immer Step by Step, langsam, langsam. Der eine lernt schneller, der andere lernt langsamer, das kommt ja noch dazu. Und die schneller lernen, die haben dann keine Geduld mehr zuzuhören, wenn die anderen sich bemühen und wollen sprechen, und da wollen die das lieber sagen, weil sie schneller sind. Das sind dann auch noch solche Herausforderungen« (LK9, Z. 254-261).

Als weiteres Problem wird die Fluktuation der VBK-Schüler*innen erlebt, da Schüler*innen über das ganze Jahr hindurch in Klassen neu zugeordnet werden, während andere aufgrund von Änderungen im Asylstatus und einem damit verbundenen Umzug die Klasse verlassen müssen. Alle Lehrkräfte sind sich einige, dass unter diesen Bedingungen das Ziel, ausreichend Deutsch zu lernen, um nach dem Ende der Vorbereitungsklasse eine Ausbildung zu beginnen, eine Illusion ist.

Hinsichtlich der Möglichkeit, die erworbenen Sprachkenntnisse in Alltagssituationen zu üben und zu festigen, sehen die befragten Lehrkräfte Defizite, die auch durch die isolierte Situation des Konstrukts >Vorbereitungsklasse $<$ hervorgebracht wird. Aus der folgenden Einlassung wird deutlich, dass Sprache als untrennbar 
mit sozialer Integration in eine Gesellschaft und als konstituierendes Element der Teilhabe an allen relevanten Lebensbereichen angesehen wird:

»Ja wie sollen sie es denn machen? Du gehst nicht auf die Straße und sagst, willst du mit mir Kontakt haben. Kontakt haben ist das Lieblingswort von einigen Schülern. Sondern das funktioniert nur im Nebeneinander und Miteinander und das schaffst du erst, deshalb ist das ja so wichtig, sie in eine Ausbildung zu bringen, in Arbeit zu bringen, denn nur dort funktioniert das wirklich. Alles andere sind aufgesetzte Sachen« (LK5, Z. 1212-1220).

Allerdings wurden die Möglichkeit für soziale Interaktion auf dem Schulgelände auch nicht konsequent genutzt, in einigen Fällen sogar eingeschränkt: Die geflüchteten Schüler*innen wurden separat von den anderen Schüler*innen unterrichtet und auf dem Schulgelände entstand eine segregierte Situation, die in einem Fall sogar in unterschiedlichen Pausenzeiten kulminierte, »um Konflikte zu vermeiden«, wie ein Schulleiter (LT2) erklärte.

Die VBK-Schüler*innen, im Rahmen einer Gruppendiskussion nach ihren Einschätzungen zum Unterrichtsangebot gefragt, äußerten sich sehr wertschätzend zu dem Sprachunterricht und beurteilten ihre eigenen Fortschritte positiv. Jedoch wünschten sie sich ein vielfältigeres Bildungsangebot, um ihre Schulbildung auch im Bereich Mathematik, Biologie oder Geschichte zu vervollständigen und auch etwas über Deutschland und Europa zu erfahren, anstatt ausschließlich Deutsch zu lernen (Abbildung. 2). Dieser Wissensbedarf verweist neben den individuellen Interessen auch auf ein Verständnis von Integration, welches nicht nur Sprache, sondern auch kulturelles Wissen umfasst. In den Wünschen der VBK Schüler*innen spiegelt sich auch der Bedarf eines konsistenten und zukunftsfähigen Wissens, ungeachtet der erlebten Brüche durch Bildungspausen und Bildungsstationen in verschiedenen nationalen Systemen. 
Abbildung 2: Visualisierung einer Gruppendiskussion mit VBK-Schüler*innen zur Evaluierung des Unterrichts

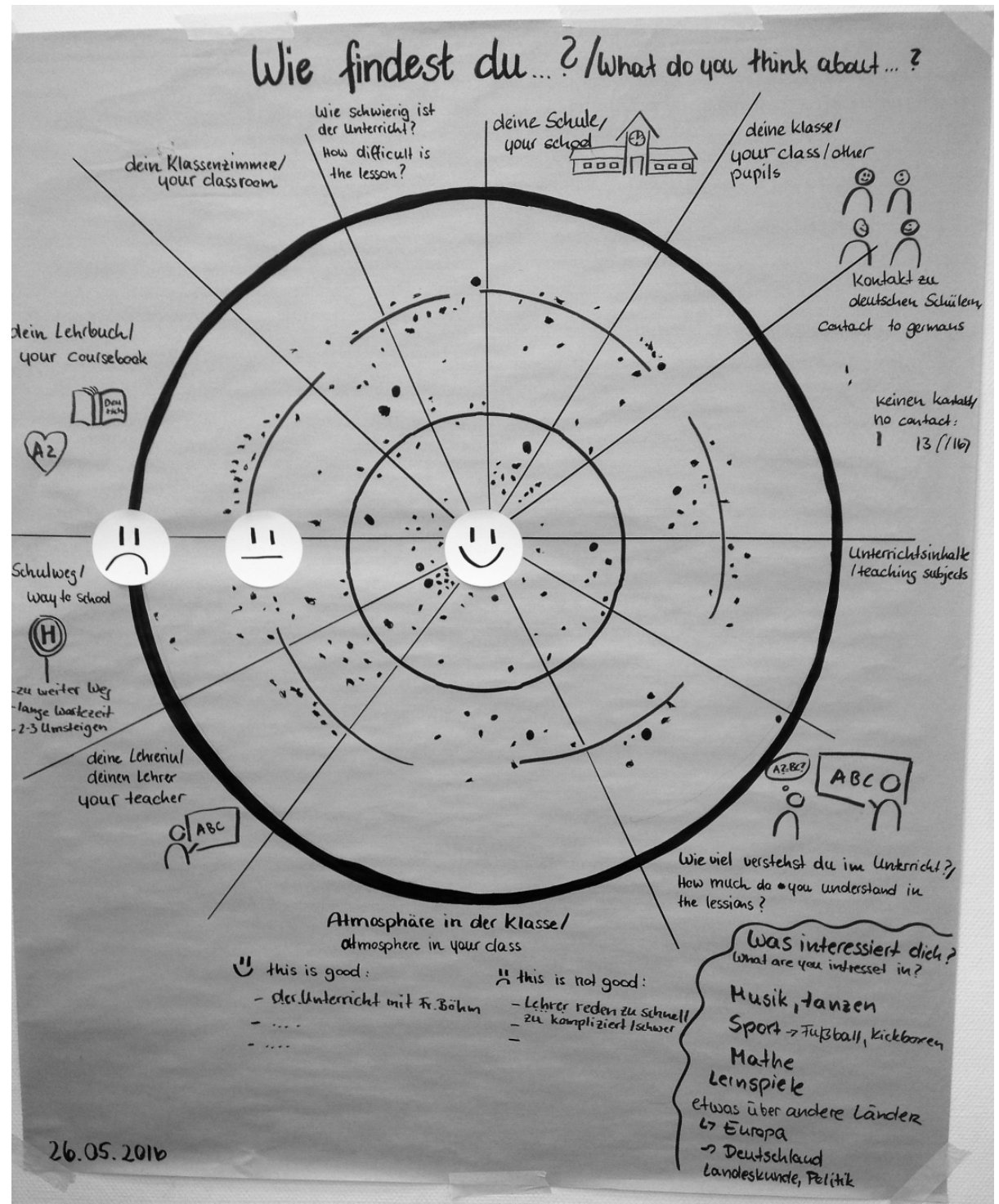

Foto: Birgit Glorius und Anne-Christin Schondelmayer, 26.5.2016 
In Bezug auf ihre alltagssprachlichen Kompetenzen außerhalb der Unterrichtssituation waren viele nicht zufrieden. Ein Schüler sagte, er würde sich aufgrund seiner schlechten Sprachkenntnisse nicht trauen, deutsche Gleichaltrige anzusprechen. $90 \%$ der befragten VBK-Schüler*innen gaben an, noch nie Kontakt zu deutschen Schüler*innen gehabt zu haben, vor allem aufgrund des getrennten Unterrichts. Den VBK-Schüler*innen war die Isolierung ihrer Gruppe sehr deutlich:

»Ein Problem ist, dass wir alle aus Afghanistan oder anderen Ländern kommen. Wir möchten Deutsch lernen mit Deutschen, weil das zum Lernen sehr gut ist. Wir möchten mit anderen Schülern zusammen mit Deutschen lernen. Das ist das Problem, ansonsten ist alles gut« (TB 1.6.2016).

Zusammenfassend kann also festgehalten werden, dass seitens der Repräsentant*innen des Bildungssystems der Spracherwerb als wesentlicher Faktor für die Integration in das deutsche Bildungs- und Ausbildungssystem und den deutschen Arbeitsmarkt betrachtet wird. Hier wird auch die eigene Zuständigkeit gesehen. Dass soziale Integration auch durch eine Vielzahl von alltäglichen Begegnungen in der Ankunftsgesellschaft hervorgebracht wird, wird zwar erkannt, jedoch werden keine Schritte ergriffen, um diese Begegnungen zu fördern. Auch die befragten geflüchteten Schüler*innen messen dem Spracherwerb einen hohen Wert bei, der sich jedoch nicht allein auf schulische Lerneinheiten bezieht, sondern auch auf notwendige und wünschenswerte Begegnungen im Alltag. Die Erweiterung des Bildungsangebots auf andere schulische Fächer ist Ausdruck des Bedürfnisses, die eigene Bildungsbiographie zu gestalten und in der Zukunft verwertbar zu machen - gleich wo diese Zukunft stattfinden wird.

\subsection{Soziale Beziehungen, Sicherheit und Stabilität}

Hinsichtlich der sozialen Beziehungen zur Mehrheitsgesellschaft stellen die Lehrkräfte der Vorbereitungsklassen die zentralen Akteur*innen dar. Da sie über Monate hinweg viel Zeit mit den Schüler*innen verbringen, wird ein teilweise enges Vertrauensverhältnis aufgebaut. Die Schüler*innen wenden sich nicht nur in Bildungsfragen an ihre Lehrkräfte, sondern auch mit Behördenschreiben, die sie nicht verstehen, und sie vertrauen den Lehrkräften auch persönliche Probleme an, etwa Sorge um Familienangehörige, die noch in den Krisengebieten leben. Aus Sicht der Lehrkräfte ist diese Beziehung an das Unterrichtsverhältnis gekoppelt, das heißt, endet die Vorbereitungsklasse, bricht auch der Kontakt ab. Eine Vielfalt an sozialen Kontakten in unterschiedlichen Lebensbereichen (z.B. Schule, 
Nachbarschaft, Sportverein) stellte sich bei den befragten Schüler*innen nicht ein.

Dies ist unter anderem auf die isolierte Situation innerhalb und außerhalb der Schule zurückzuführen, in der sich die Schüler*innen befinden. Während es auf dem Schulcampus kaum Begegnungsmöglichkeiten mit den anderen, deutschsprachigen Schüler*innen gibt, trägt die vielfach isolierte und periphere Wohnsituation in den umliegenden Gemeinden weiter zur Exklusion bei. So ist der Alltag der VBK-Schüler*innen außerhalb der Schule geprägt durch soziale Isolation aufgrund von schlechter Mobilitätsinfrastruktur in der ländlichen Region, fehlender Diversität und einem Mangel an zielgruppenspezifischen Beratungs- und Unterstützungsangeboten. Gerade Schüler*innen, die ohne Angehörige nach Deutschland gekommen sind, werden durch diese isolierte Lebenssituation stark belastet und versuchen, durch einen Umzug in größere Städte ihre Lebenslage zu verbessern, wie der folgende Protokollauszug zeigt:

»Im Gespräch mit A. erzählt er mir, dass er sich freut, dass er morgen nach S.-Stadt umzieht. O.-Stadt gefällt ihm nicht, da er kein Fahrticket habe und die Kleinstadt sehr begrenzt sei. In S.-Stadt würde er mit seinem Freund zusammen sein, der auch aus Syrien kommt. Hier in O.-Stadt sei er ganz allein. Mit dem Umzug verspricht A. sich auch, öfter dann nach C.Stadt zu fahren, denn er habe dann ja die Möglichkeit. Die Stadt gefällt ihm sehr gut und er habe da über Freunde die Möglichkeit in einem Restaurant zu arbeiten, da er arabisch spricht und das Restaurant nach einem arabisch sprechenden Mitarbeitenden suche« (TB 1.6.2016).

Ein weiteres Hindernis für die soziale Integration vor Ort sind die Erfahrungen der VBK-Schüler*innen mit Fremdenfeindlichkeit und Rassismus. Sie berichten von Beleidigungen, wenn sie mit dem öffentlichen Bus unterwegs sind und auch von tätlichen Angriffen. Mehrfach hatte es Anschläge auf die Gruppenunterkünfte oder Privatwohnungen der Schüler*innen gegeben, die nicht aufgeklärt werden konnten. Dabei fühlten sich die Betroffenen von den deutschen Behörden allein gelassen, wie dieser Schüler erzählt:

»Vor zwei Monaten hat ein Mann einen Stein genommen und hat in unsere Fenster [...] einfach so: zum Spaß [...] wir haben die Polizei angerufen //ja// aber dann hat die Polizei dreißig Minuten später gekommen weil ist Polizisten von A.-Stadt $/ / \mathrm{mmh} / /$ gibt's keine Polizei in E.-Stadt [...] und waren da und haben gesagt wir können nichts machen [...] weil war betrunken er weiß nicht was macht und ich-ich wollte wa- bisschen was konnte ich sagen //mmh// weil war ein Schock für mich« (SL1, Z.642-648).

Nach einem weiteren tätlichen Übergriff wandte sich die Familie an den Bürgermeister des Ortes mit der Bitte um Unterstützung. Dieser schlug vor, die Familie in einen anderen Ort umzusiedeln: 
»Wir sind zum Rathaus gegangen und mit dem Bürgermeister geredet //ja// und hat gesagt okay wir können machen ein Transfer in eine andere Stadt //ja// er kann nicht besser machen und wo hat gesagt wir müssen S.-Stadt gehen wo er wohnt //ja// und wir haben gesagt nein wenn wir kennen Leute darüber //ja// wir sind schon ein Jahr da $/ / \mathrm{mmh} / /$ und wir können nicht einfach noch einmal anfangen« (SL1, Z.693-698).

Dieses Erlebnis steht stellvertretend für die vielfachen Berichte über ablehnendes und fremdenfeindliches Verhalten der lokalen Bevölkerung. Erschwerend kommt die starke Politisierung des Themas Flucht und Asyl ab dem Jahr 2015 hinzu, was vor allem in Sachsen zu einer stark erhöhten Zahl fremdenfeindlicher Aktivitäten, rechtsextremistischer Mobilisierung und entsprechenden politischen Wahlentscheidungen führte.

Die fremden- und asylfeindliche Stimmung wird seitens der untersuchten Schulen still akzeptiert. Lehrer*innen, die sich innerhalb des Schulkollegiums (erfolgreich) weigerten, Ausländer*innen zu unterrichten, wurden nicht sanktioniert. Eine >Deutsch als Zweitsprache<-Lehrerin berichtete von Vorbehalten aus ihrem eigenen Bekanntenkreis hinsichtlich ihrer Tätigkeit sowie von eigenen Erlebnissen mit fremdenfeindlichen Reaktionen gegenüber ihren Schüler*innen:

»Ich gehe auch mit großen Gruppen raus, mache Exkursionen auch wenn man mal im Bus oder im Zug und überall auffällt auch manchmal aneckt, die Schüler tun ja nichts, aber wenn ich eben mit einer Gruppe komme, und da sind zehn von schwarz, die total auffallen, dann trifft man eben auf das normale Leben hier und wird eben auch manchmal beleidigt oder beschimpft« (LK5, Z. 930-935)

Das Zitat zeigt, dass fremdenfeindliche Äußerungen im Sozialraum als normal und unabänderlich wahrgenommen werden (»das normale Leben«). Auf diese Weise stehen die VBK-Schüler*innen mit ihren Erfahrungen von Ablehnung allein, was zu einer zusätzlichen Isolation und dem Gefühl von Unsicherheit führt. So berichteten einige Schüler*innen, dass sie sich nur innerhalb des Klassenraums wohl fühlten, und dass sie sich im öffentlichen Raum aus Angst vor Pöbeleien und Angriffen nur in Gruppen bewegten. Nimmt man zu diesen Erfahrungen noch die allgemeine Unsicherheit hinsichtlich des Ausgangs des Asylverfahrens und seiner Konsequenzen hinzu, kommt man zu dem Schluss, dass ein wichtiger »weicher Faktor« von Integration in dem vorgestellten Fallbeispiel nicht gegeben ist.

\section{Diskussion und Fazit}

In unserem Beitrag sind wir anhand einer konkreten Fallstudie zur Bildungsintegration von Geflüchteten im berufsbildenden Bereich der Frage nachgegangen, 
welche Perspektiven auf Integration seitens der wichtigsten beteiligten Akteur*innengruppen geäußert werden und damit handlungsleitend sind. Damit fokussieren wir auf das Handeln von Akteur*innen in einer Organisation und letztlich darauf, wer sich für welche Schritte verantwortlich fühlt (oder auch nicht) sowie darauf, wer welchen Handlungsspielraum erkennt und zur Verfügung hat. Im Folgenden sollen die gefundenen Perspektiven in generalisierter Form dargestellt und ihre praktischen Konsequenzen für den Integrationsprozess sowie denkbare Gegenhorizonte aufgezeigt werden.

Die erste dominante Perspektive auf Integration war die systemische und formale Perspektive, das heißt die Integration der Vorbereitungsklassen in das Gesamtsystem der Beruflichen Schulzentren. Diese Perspektive wurde vor allem vom Leitungspersonal und von Vertreter*innen der Bildungsbehörden eingenommen. In der Handlungspraxis lag dementsprechend der Fokus auf organisatorischen Aufgaben und verhinderte die Thematisierung gestalterischer Fragen, die eine langfristige Integration der geflüchteten Schüler*innen und mögliche Wege der Integration in den Blick nehmen, wie beispielsweise Praktikumsangebote oder weitere Beschäftigungsmaßnahmen vor Ausbildungsbeginn. Diese Lesart von Integration bewegt sich innerhalb der sektoralen und institutionellen Grenzen, mit denen sich die jeweiligen Akteur*innen in ihrer professionellen Rolle identifizieren. Ein möglicher Gegenhorizont wäre durch eine Perspektive gegeben, in der die individuelle Bildungsbiographie im Mittelpunkt steht und die handelnden Akteur*innen über Ressortgrenzen hinweg zusammenwirken, um diese Biographie bestmöglich zu gestalten. Auf der Systemebene würde dies bedeuten, bereits etablierte Programme des sogenannten Übergangssystems wie das Berufsvorbereitungsjahr oder die Einstiegsqualifikation zu einem Standardangebot auch für geflüchtete Schüler*innen im höheren Bildungsalter zu machen.

Eine zweite dominante Lesart von Integration, die in der Studie aufscheint, ist jene von der angenommenen Einseitigkeit des Integrationsprozesses, der darauf ausgerichtet ist, die Vorbereitungsklassen in das System Berufsschule und die Schüler*innen der Vorbereitungsklassen in das deutsche Bildungssystem und den deutschen Arbeitsmarkt zu integrieren. Die angebotenen Maßnahmen werden von der Anbieterseite definiert, die VBK-Schüler*innen müssen die Angebote annehmen, ungeachtet ihrer unterschiedlichen Herkunft, ihres Bildungsstandes und ihrer Bildungsaspirationen. Dies entspricht einer Lesart von Integration, welche allein an Migrant*innen orientiert ist, die es in ein bestehendes System bzw. Teilsysteme einzugliedern gilt und fokussiert auf eine strukturelle und messbare Ebene von Teilhabe. Ein möglicher Gegenhorizont in Form eines inklusiven Bildungsverständnisses würde sich - wiederum basierend auf der individuellen 
Betrachtung des Einzelfalls - auf die Generierung bzw. Vermittlung von Kenntnissen und Fähigkeiten konzentrieren, die für die weitere Gestaltung der individuellen Bildungsbiographie nützlich sind und dabei die Vielfalt der Vorkenntnisse und Fähigkeiten berücksichtigen. Dies würde zugleich auch notwendige Anpassungsleistungen des (Bildungs-)Systems implizieren.

Ein drittes Charakteristikum an der vorgefundenen Auslegung des Integrationsbegriffs ist die Missachtung von Kontextbedingungen, insbesondere was die soziale Integration im Alltag anbelangt. In dem geschilderten Fallbeispiel hat dies zur Konsequenz, dass die VBK-Schüler*innen mit einer gleichgültigen bis ablehnenden (und zum Teil sogar gefährlichen) Alltagsumgebung allein gelassen werden. Das für Integrationsprozesse notwendige Kriterium der Sicherheit und Stabilität des eigenen Umfeldes und der vielfältigen und freundlich gesinnten Kontakte zur Mehrheitsgesellschaft ist damit nicht gegeben. Unter diesen Umständen ist nicht davon auszugehen, dass die jugendlichen Geflüchteten länger als formal notwendig in den zugewiesenen Gemeinden verbleiben werden. Ein möglicher Gegenhorizont wäre hier, dass auch die soziale Integration im Alltag von den handelnden Akteur*innen vor Ort als gemeinsame Aufgabe begriffen wird. Dies würde die Bildung von ressort- und institutionenübergreifenden Akteur*innennetzwerken beinhalten, eine Sensibilisierung und Stärkung der Zivilgesellschaft vor Ort sowie die Installation von kompetenten Beratungs- und Unterstützungsmechanismen für Integration, gegen Ausgrenzung und Rassismus und ebenso die Berücksichtigung der Interessen der Neuzugewanderten sowie Partizipationsmöglichkeiten.

Viele der hier dargestellten Befunde bestätigen Erkenntnisse anderer Studien zur Bildungsintegration, etwa das Ergebnis der mangelnden Passfähigkeit des vorgehaltenen Bildungsangebots mit den Wünschen, Bildungs- und Lebensperspektiven der Angebotsnutzer*innen, das vor allem durch eine pure Übertragung von Konzepten aus anderen Bildungsbereichen resultiert (vgl. Abschnitt 2.3). Der Fokus auf einen intensiven Sprachlehrgang, der in den Vorbereitungsklassen eingenommen wird, entstammt der Programmatik der Integration in allgemeinbildende Schulen, in denen sich allerdings an den Besuch der Vorbereitungsklasse eine graduelle Integration in die reguläre Klassenstruktur anschließt. Die Übertragung dieses Konzepts auf das System Berufsschule scheitert an der völlig anderen Strukturierung von berufsschulischen Fachklassen, sowie durch die am Lebensalter festgemachten Restriktionen der Teilhabe, da mit Erreichen der Volljährigkeit das von uns untersuchte Bildungsangebot von den Geflüchteten nicht mehr wahrgenommen werden kann. 
Auch die heterogene Bildungserfahrung der Lerngruppen geflüchteter Schüler*innen und die Schwierigkeiten, die dies für den Unterrichtsalltag mit sich bringt, sind in unserer Studie deckungsgleich zu oben diskutierten Studien. Die Möglichkeit, durch differenzierte Einstufungstests und das Vorhalten von unterschiedlichen Leistungsstufen im Bereich Vorbereitungsklassen auf unterschiedliches Vorwissen und Lerngeschwindigkeiten einzugehen, ist letztlich eine Frage der Verfügbarkeit. In dem von uns untersuchten Beispiel stellen die ländlich-periphere Struktur des Untersuchungsraums, der allgemeine Mangel an qualifiziertem Unterrichtspersonal sowie die relativ geringe Anzahl an geflüchteten Schüler*innen objektive Hinderungsgründe für eine weitere Ausdifferenzierung des Unterrichtsangebots dar. Auch die Überlagerung von schulischen und aufenthaltsrechtlichen Bestimmungen und Zuständigkeiten, die in anderen Studien thematisiert wurde, lässt sich in unserem Beispiel als zusätzliches Hindernis auf dem Weg zu einer nachhaltigen Integration auffinden.

Anders als in vielen der oben diskutierten Studien hat unsere Studie nicht ein implizites gemeinsames Verständnis von Integration aufseiten der handelnden Akteur*innen angenommen, sondern hat genau dieses Verständnis in das Zentrum der Untersuchung gestellt. Diese offene Herangehensweise und die Konzentration auf die Wissensbestände und Handlungspraktiken der Akteur*innen vor Ort sowie der ganzheitliche Blick auf das Feld in seiner raumzeitlichen Einbettung haben es ermöglicht, faktische und subjektiv empfundene Problemfelder, aber auch Handlungspotenziale aufzuspüren. Zugleich konnten wir durch unsere Vorgehensweise deutlich machen, dass viele der aufgezeigten Probleme nicht der Spezifik der betrachteten Gruppe der Geflüchteten inhärent sind, sondern strukturell bzw. systemspezifisch sind, wie etwa die periphere Lage des Landkreises, Mangel an qualifiziertem Lehrpersonal, ressortzentrierte Problemwahrnehmungen oder auch die mangelnde Offenheit des Systems Schule gegenüber heterogener Lerngruppen und Bedürfnisse. Die vermehrte Ankunft von Geflüchteten könnte dementsprechend als Ausgangspunkt genommen werden, um grundsätzliche Veränderungen oder auch Innovationen in dem betrachteten System anzugehen.

Zudem wäre eine breite gesellschaftliche Debatte darüber, was Staat und Gesellschaft von Zugewanderten (nicht nur von Geflüchteten) konkret erwarten und was man dafür auch zu geben und zu ändern bereit ist, für eine zukunftsweisende Entwicklung von Integrationswegen in das Bildungs- und Ausbildungssystems wünschenswert. 


\section{Literatur}

Ager, Alastair/Strang, Alison (2008), Understanding Integration: A Conceptual Framework, Journal of Refugee Studies, 21 (2), 166-191.

Auernheimer, Georg (Hrsg.) (2013), Schieflagen im Bildungssystem. Die Benachteiligung der Migrantenkinder, Wiesbaden.

Autorengruppe Bildungsberichterstattung (ABB) (2016), Bildung in Deutschland 2016. Ein indikatorengestützter Bericht mit einer Analyse zu Bildung und Migration, Bielefeld.

Baethge, Martin/Seeber, Susan (2016), Herausforderungen der Flüchtlingsmigration für die Strukturen beruflicher Bildung in Deutschland. Expertise im Auftrag des Sachverständigenrats deutscher Stiftungen für Integration und Migration für das Jahresgutachten 2017, Göttingen.

Bauer, Angela/Schreyer, Franziska (2016), Ausbildung von unbegleiteten minderjährigen Flüchtlingen: Sinnvoll ist Unterstützung über Volljährigkeit hinaus, IAB-Kurzbericht, No. 13/2016.

Baur, Christine/Häussermann, Hartmut (2009), Ethnische Segregation in deutschen Schulen, Leviathan, 37, 353-366.

Bohnsack, Ralf (2014), Rekonstruktive Sozialforschung. Einführung in Methodologie und Praxis qualitativer Forschung, Opladen.

Bohnsack, Ralf (2017), Konjunktiver Erfahrungsraum, Regel und Organisation, in: Amling, Steffen/Vogd, Werner (Hrsg.), Dokumentarische Organisationsforschung. Perspektiven der praxeologischen Wissenssoziologie, Leverkusen, 229-255.

Bourdieu, Pierre (1986), The Forms of Capital, in: Richardson, John E. (Hrsg.), Handbook of Theory of Research for the Sociology of Education, Westport, 241-258.

Bowskill, Matt/Lyons, Evanthia/Coyle, Adrian (2007), The Rhetoric of Acculturation: When Integration Means Assimilation, British Journal of Social Psychology, 46 (4), 793-813.

Bundesamt für Migration und Flüchtlinge (BAMF) (Hrsg.) (2015), Das Bundesamt in Zahlen 2014. Asyl, Migration und Integration, Nürnberg.

Bundesamt für Migration und Flüchtlinge (BAMF) (Hrsg.) (2016), Das Bundesamt in Zahlen 2015. Asyl, Migration und Integration, Nürnberg.

Bundesamt für Migration und Flüchtlinge (BAMF) (Hrsg.) (2017), Aktuelle Zahlen zu Asyl, Dezember 2016, Nürnberg.

Bundesamt für Migration und Flüchtlinge (BAMF) (Hrsg.) (2018), Aktuelle Zahlen zu Asyl, Dezember 2017, Nürnberg. 
Bundesinstitut für Berufsbildung (BIBB) (Hrsg.) (2017), Datenreport zum Berufsbildungsbericht 2017. Informationen und Analysen zur Entwicklung der beruflichen Bildung, Bonn.

Crul, Maurice/Schneider, Jens (2010), Comparative Integration Context Theory: Participation and Belonging in New Diverse European Cities, Ethnic and Racial Studies, 33 (7), 1249-1268.

Der Sächsische Ausländerbeauftragte (DSA) (Hrsg.) (2018), Jahresbericht 2017 des Sächsischen Ausländerbeauftragten, Sächsischer Landtag, 6. Legislaturperiode, Dresden.

Dietz, Martin/Trübswetter, Parvati (2016), Early Intervention - Teilnehmerstruktur und Arbeitsmarktintegration von Asylbewerberinnen und Asylbewerbern, Berufsbildung in Wissenschaft und Praxis, 45 (1), 4-5.

El-Mafaalani Aladin (2016), Diskriminierung von Menschen mit Migrationshintergrund, in: Scherr, Alfred/El-Mafaalani Aladin/Gökcen Yüksel, Emine (Hrsg.), Handbuch Diskriminierung, Wiesbaden, 1-14.

Esser, Hartmut (2000), Soziologie. Spezielle Grundlagen, Band 2: Die Konstruktion der Gesellschaft, Frankfurt/M.

Foroutan, Naika (2015), Unity in Diversity. Integration in a Post-Migrant Society. Policy Brief. Bundeszentrale für politische Bildung. http://www.bpb.de/gesells chaft/migration/kurzdossiers/205290/integration-in-a-post-migrant-society, 22.2.2018.

Gag, Maren/Schroeder, Joachim (2014), Monitoring und Bildungsberichterstattung mit Fokus auf Flüchtlinge und Asylsuchende - ein Beispiel, in: Gag, Maren/Voges, Franziska (Hrsg.), Inklusion auf Raten. Zur Teilhabe von Flüchtlingen an Ausbildung und Arbeit, Münster, 29-48.

Glorius, Birgit (2014), Bildungsbenachteiligung durch Migration? Kinder und Jugendliche mit Migrationshintergrund im deutschen Bildungssystem, in: Gans, Paul (Hrsg.), Räumliche Auswirkungen der internationalen Migration, Hannover, 178-199.

Georgi, Viola (2015), Integration, Diversity, Inklusion. Anmerkungen zu aktuellen Debatten in der deutschen Migrationsgesellschaft, DIE Zeitschrift für Erwachsenenbildung, II/2015, 25-27.

Gogolin, Ingrid (2013), Mehrsprachigkeit, Stichwort: Zeitschrift für Erziehungswissenschaft, Wiesbaden, 340-358.

Gomolla, Mechthild/Radtke, Frank-Olaf (2009), Institutionelle Diskriminierung. Die Herstellung ethnischer Differenz in der Schule, Wiesbaden. 
Gurski, Lara/Rother Timo (2018), Wie erleben geflüchtete Jugendliche Schule in verschiedenen Lerngruppen? Erfahrungsberichte von inkludierten und (noch) nicht inkludierten Flüchtlingen, in Große Prues, Peter (Hrsg.), Inklusion im Rahmen von Schule - eine Aufgabe, viele Möglichkeiten. Ein Sammelband von Studierenden der Universität Osnabrück, Osnabrück, 173-184.

Heinrichs, Karin, et al. (2016), Die Implementierung neuer Konzepte zur Beschulung von Flüchtlingen und Asylsuchenden - Herausforderung und Chancen aus organisations- theoretischer Perspektive, Gruppe, Interaktion, Organisation, 47 (3), 231-241.

Hersi, Abidi M. (2014), Discourses Concerning Immigrant Integration: A Critical Review, European Scientific Journal, 1857-7881.

Kelch, Manja (2016), Sachsens Schulen nehmen immer mehr Flüchtlinge auf, http://www.bildung.sachsen.de/blog/index.php/2016/03/22/sachsens-schulen-n ehmen-immer-mehr-fluechtlinge-auf/, 30.12.2018.

Kemper, Thomas (2017), Bildungsbeteiligung von Schülerinnen und Schülern mit Migrationshintergrund in Abhängigkeit von der schulstatistischen Operationalisierung, Die deutsche Schule, 109 (1), 91-115.

Lockwood, David (1964), Social Integration and System Integration, in: Zollschan, Georg K./Hirsch, Walter (Hrsg.), Explorations in Social Change, London, 244-257.

Mannheim, Karl (1964), Wissenssoziologie, Neuwied.

Mercator-Institut für Sprachförderung und Deutsch als Zweitsprache und Zentrum für LehrerInnenbildung der Universität zu Köln (Hrsg.) (2015), Neu zugewanderte Kinder und Jugendliche im deutschen Schulsystem. Bestandsaufnahme und Empfehlungen. http://www.mercator-institut-sprachfoerderung.de/fileadm in/Redaktion/PDF/Publikationen/MI_ZfL_Studie_Zugewanderte_im_deutsche n_Schulsystem_final_screen.pdf, 15.9.2016.

Metzner, Franka, et al. (2018), Soziale Unterstützung bei unbegleitet und begleitet geflüchteten Jugendlichen und jungen Erwachsenen nach der Ankunft in Deutschland: Ergebnisse einer Befragung in >Willkommensklassen burger Berufsschulen, Zeitschrift für Flüchtlingsforschung, 2 (1), 3-31.

Meyer, Frauke (2014), »Das ist für uns schon ein Experiment" - Erfahrungen von Ausbilderinnen und Ausbildern mit jungen Flüchtlingen in der dualen Ausbildung, https://www.fluchtort-hamburg.de/fileadmin/pdf/2015/passage_FOH_2 014_Broschuere_Web.pdf, 14.8.2019.

Nohl, Arnd-Michael (2010), Konzepte interkultureller Pädagogik. Eine systemische Einführung, Bad Heilbrunn. 
Nohl, Arnd-Michael (2017), Organisationen in der dokumentarischen Mehrebenenanalyse, in: Amling Steffen/Vogd, Werner (Hrsg.), Dokumentarische Organisationsforschung. Perspektiven der praxeologischen Wissenssoziologie, Leverkusen, 279-300.

Przyborski, Aglaja/Wohlrab-Sahr, Monika (2014), Qualitative Sozialforschung. Ein Arbeitsbuch, München.

Putnam, Robert (1993), The Prosperous Community: Social Capital and Public Life, American Prospect, 13, 35-42.

Reich, Hans/Roth, Hans-Joachim (2002), Spracherwerb zweisprachig aufwachsender Kinder und Jugendlicher - Ein Überblick über den Stand der nationalen und internationalen Forschung. Hamburg. Verfügbar unter: www.ew.unih amburg.de/ueber-die-fakultaet/personen/neumann/files/gutachten.pdf, 27.9.2019.

Riegel, Christine (2009), Integration - ein Schlagwort? Zum Umgang mit einem problematischen Begriff, in: Sauer, Karin E./Held, Josef (Hrsg.), Wege der Integration in heterogenen Gesellschaften. Vergleichende Studien, Wiesbaden, 23-40.

Robinson, Vaughan (1998), Defining and Measuring Successful Refugee Integration, Proceedings of ECRE International Conference on Integration of Refugees in Europe, Antwerp, November 1998, Brüssel.

Sächsisches Staatsministerium für Kultus (SMK) (2000), Die sächsische Konzeption zur Integration von Migranten. Ministerialblatt des Sächsischen Staatsministeriums für Kultus, 2000/8, https://www.revosax.sachsen.de/vorschrift/9651 -Konzeption-zur-Integration-von-Migranten\#x 1, 1.1.2019.

Sächsisches Staatsministerium für Kultus (SMK) (2016), Jugendliche mit Migrationshintergrund in Vorbereitungsklassen an Beruflichen Schulzentren, Rundbrief vom 7.3.2016, Dresden.

Scheiermann, Gero/Walter, Marcel (2016), Flüchtlingsintegration durch berufliche Bildung - neue Herausforderungen und improvisierte Lösungen in einem alten Handlungsfeld, Berufs- und Wirtschaftspädagogik-online, Nr. 30, http:// www.bwpat.de/ausgabe30/scheiermann_walter_bwpat30.pdf, 14.8.2019.

Speer, Marc/Klaus, Tobias (2015), Der lange Weg zur Arbeit. Qualitative Studie zu räumlichen Möglichkeiten und Hindernissen einer Partizipation von Flüchtlingen am Arbeitsmarkt am Beispiel von vier Standorten in Niederbayern, https://www.fluechtlingsrat-bayern.de/tl_files/PDF-Dokumente/Der\%201a nge $\% 20 \mathrm{Weg} \% 20$ zur\%20 Arbeit $\% 20$ bfr-Bleib\%20in\%20Bayern.pdf, 14.8.2019. 
Spindler, Susanne (Hrsg.) (2016), Geflüchtete Kinder und Jugendliche im Bildungssystem. Veröffentlicht am 25.7.2016 in socialnet-Materialien unter http:// www.socialnet.de/materialien/27589.php, 4.10.2019.

Schroeder, Christoph/Gornitzka, Lydia/Steinbock, Dorothee (2015), Bildungszugang und Deutscherwerb für Flüchtlinge in Deutschland, Expertise im Auftrag der Robert Bosch Stiftung, Potsdam. https://www.bosch-stiftung.de/sites/defau lt/files/publications/pdf_import/Kommissionsbericht_Fluechtlingspolitik_Bild ung.pdf, 14.8.2018.

Studnitz, Stefanie (2011), Ausgrenzung statt Ausbildung - die Situation junger Flüchtlinge im deutschen Bildungssystem, Migration und Soziale Arbeit, 33(2), 130-136.

Vossenkuhl, Andreas (2010), (Berufs-)Schulpflicht in Deutschland, $B O B B, B W P$ 6/2010, https://www.bibb.de/veroeffentlichungen/en/publication/download/65 $12,1.1 .2019$.

UNICEF (2016), Zur Situation der Flüchtlingskinder in Deutschland. UNICEFLagebericht, Köln. https://www.unicef.de/blob/115186/de54a5d3a8b6ea03337 b489816eeaa08/zur-situation-der-fluechtlingskinder-in-deutschland-data.pdf, 1.1.2019.

vbw - Vereinigung der Bayerischen Wirtschaft (Hrsg.) (2016), Integration durch Bildung. Migranten und Flüchtlinge in Deutschland. Gutachten. Münster.

Vogel, Dita/Stock, Elina (2017), Opportunities and Hope Through Education: How German Schools Include Refugees, Brüssel. http://www.fb12.uni-bremen .de/fileadmin/Arbeitsgebiete/interkult/Vogel/17_Vogel_Stock_Refugee_Scho ols_Germany.pdf, 14.8.2019

Wirtschaftsförderung Erzgebirge GmbH (2014), Standortprofil Erzgebirge/Erzgebirgskreis, Annaberg-Buchholz.

\section{Autorinnen}

Birgit Glorius, Prof. Dr., Institut für Europäische Studien und Geschichtswissenschaften, TU Chemnitz

Anne-Christin Schondelmayer, Prof. Dr., Institut für Allgemeine Erziehungswissenschaft, Universität Koblenz-Landau 